\title{
Metástase gástrica com sitio primário em mama: relato de caso
}

\author{
Gastric metastasis with mamary breats site: case report
}

\author{
Metastasis gástrica con sitio primario en mama: reporte de caso
}

Laura Loth Marton Azzi ${ }^{1 *}$, Matheus Silva Costa Corrêa Alves ${ }^{1}$, Pedro Icaro Navarro Sapori ${ }^{1}$, Lídia Carneiro de Sousa1, Luisa Cunha Guimarães ${ }^{1}$, Luiza Passos Ribeiro', Nina Vasconcelos Pessanha², Leonardo Salviano da Fonseca Rezende², Gabriel Mazoni Silva Martins².

\section{RESUMO}

Objetivo: O objetivo principal desde artigo é relatar o caso de uma paciente com caso prévio de câncer de mama e mastectomia que após 3 anos que apresentou metástase gástrica na forma de linite plástica. Tem como objetivo secundário atentar profissionais da saúde quanto a relação entre disseminação de carcinoma mamário para estômago causando sintomas dispépticos e/ou obstrutivos. Detalhamento de caso: Paciente do sexo feminino, de 51 anos, com história pregressa de carcinoma ductal invasor de mama, com queixa de sintomas de abdome agudo obstrutivo por linite plástica (BORMANN 4) cujo tumor primário era de proveniente da mama. O diagnóstico definitivo foi possível apenas pela análise imuno-histoquímica, a qual tem se mostrado de grande valor neste tipo de caso. Considerações finais: Devido à grande morbimortalidade dessa patologia deve-se descartá-la em casos de sintomas gastrointestinais em pacientes com quadros prévios de neoplasia maligna mamária em que outras causas mais comuns não podem ser encontradas.

Palavras-chave: Neoplasia, Mama, Linite plástica, Metástase, Gastrointestinal.

\begin{abstract}
Objective: The main objective of this article is to report the case of a patient with a previous case of breast cancer and mastectomy who, after 3 years, had gastric metastasis in the form of plastic linitis. Its secondary objective is to assist health professionals regarding the relationship between the spread of breast carcinoma to the stomach causing dyspeptic and / or obstructive symptoms. Case details: Female patient, 51 years old, with a past history of invasive breast ductal carcinoma, complaining of symptoms of acute obstructive abdomen due to plastic linitis (BORMANN 4) whose primary tumor was from the breast. The definitive diagnosis was made possible only by immunohistochemical analysis, which has proved to be of great value in this type of case. Final considerations: Due to the great morbidity and mortality of this pathology, it should be ruled out in cases of gastrointestinal symptoms in patients with previous cases of malignant breast cancer in which other more common causes cannot be found.
\end{abstract}

Keywords: Neoplasia, Breast, Linitis plastica, Metastasis, Gastrointestinal.

\section{RESUMEN}

Objetivo: El objetivo principal de este artículo es reportar el caso de una paciente con un caso previo de cáncer de mama y mastectomía que, a los 3 años, presentó metástasis gástrica en forma de linitis plástica. Su objetivo secundario es asistir a los profesionales de la salud en la relación entre la diseminación del carcinoma de mama al estómago causando síntomas dispépticos y / u obstructivos. Detalles del caso: Paciente de sexo femenino, 51 años, con antecedente de carcinoma ductal de mama invasivo, que consulta por cuadro de abdomen agudo obstructivo por linitis plástica (BORMANN 4) cuyo tumor primario era de mama. El diagnóstico definitivo sólo fue posible mediante el análisis inmunohistoquímico, que ha demostrado ser de gran valor en este tipo de casos. Consideraciones finales: Debido a la gran morbimortalidad de esta patología, conviene descartarla en casos de síntomas gastrointestinales en pacientes con casos previos de cáncer de mama maligno en los que no se puedan encontrar otras causas más frecuentes.

Palabras clave: Neoplasia, Mama, Linitis plástica, Metástasis, Gastrointestinal.

${ }^{1}$ Faculdade de Minas (FAMINAS), Belo Horizonte - MG. *E-mail: lauraazzi@hotmail.com

${ }^{2}$ Santa Casa de Misericórdia de Belo Horizonte, Belo Horizonte - MG. 


\section{INTRODUÇÃO}

A neoplasia maligna de mama é o tumor mais incidente no sexo feminino, com exceção apenas do câncer de pele não-melanoma, cuja suas principais metástases para ossos, pulmão, fígado e cérebro. Entretanto o sítio gástrico é um local incomum para disseminação desse tumor (ABID A, et al., 2013).

A neoplasia maligna da mama quando apresenta metástases gástrica é um diagnóstico raro, ocorrendo em menos de $1 \%$ dos casos de acordo com a literatura. Isso torna o diagnóstico muito difícil, levando um tempo médio entre o diagnóstico do tumor de mama até a metástase gástrica em torno de 4 a 10 anos (JONES GE, et al., 2007). Por tratar-se de um tempo relevante, a maioria dos sítios metastáticos é diagnosticado em estágios mais avançados, no qual as medidas de tratamento podem não ser efetivas, além de não modificarem o curso da doença, tornando a proposta curativa não viável. Neste contexto, os cuidados paliativos ganham uma importante relevância com o objetivo de oferecer uma melhor qualidade de vida para os doentes (LIMA LC, et al., 2018).

A forma mais comum de metástase gástrica do câncer de mama é o acometimento na forma de linite plástica, um adenocarcinoma gástrico, infiltrativo e difuso. Na grande maioria, $83 \%$ dos casos, observados em autopsias, o câncer de mama primário que levava a um tumor gástrico era um carcinoma do tipo lobular invasivo, sendo ainda mais raro em casos de carcinoma ductal invasor, o tipo histológico mais comum (HARA F, et al., 2010).

Os sintomas mais frequentes são epigastralgia, dispepsia, anorexia, náuseas e vômitos, sendo a presença de massa endurecida palpável em epigástrio, o sinal mais comum em pacientes com essa patologia. Contudo, a presença de sinais e sintomas inespecíficos acabam retardando o diagnóstico da doença. Estima-se que $6 \%$ das pacientes com câncer de mama apresentem acometimento gástrico, embora esse valor chegue a 18\% em autopsias (CIULLA A, et al., 2008).

A propedêutica diagnóstica inclui endoscopia digestiva alta (EDA) com biópsia e tomografia computadorizada (TC), entretanto, mesmo com a presença de exames de imagem com achados sugestivos de câncer, a confirmação do diagnóstico através de exames histológicos e imuno-histoquímicos se faz necessária (ARAÚJO LH, et al., 2007).

A TC é o exame de escolha para o estadiamento, tanto de abdome quanto de tórax. A ressonância magnética é indicada na propedêutica para metástases hepáticas, sendo ainda um exame pouco disponível no serviço de saúde pública (ARAÚJO LH, et al., 2007).

A imuno-histoquímica, incluindo pesquisa de receptores hormonais também pode ser útil nessa investigação. A presença de citoqueratinas, como a CK20, de baixo peso molecular e a ausência de outros tipos, como a CK7, sugerem fortemente tumores do cólon. O padrão inverso, todavia, sugerem origens como pulmão, mama, ovário. Isso ocorre, porque tais citoqueratinas são expressas pelos órgãos de origem do tumor (ARAÚJO LH, et al., 2007).

A pesquisa de receptores de estrôgenio e progesterona apresenta também grande especificidade diagnóstica. As citoqueratinas são proteínas de queratina que compõem filamentos intermediários encontrados no citoesqueleto intracitoplasmático do tecido epitelial, possuindo diversos tipos. A ausência da citoqueratina CK20 e a presença da CK7 favorecem o diagnóstico de tumor metastático de mama. A proteína do receptor do fator de crescimento epitelial humano conhecido como receptor HER2 não fornece bom parâmetro de confirmação, mas indica um prognóstico reservado, com necessidade do uso de trastuzumabe (ARAÚJO LH, et al., 2007; JUCA PC, 2016).

Levando em consideração o tratamento dos pacientes com acometimento do trato gastrointestinal temos como opção terapêutica a quimioterapia e/ou terapia hormonal. A remoção do estômago é indicada apenas no contexto de doença primária controlada e ausência de outros sítios metastáticos. Ainda assim, a sobrevida esperada varia de 7 a 38 meses (RODRIGUES MV, et al., 2016; LIBÂNIO D, et al., 2018).

O objetivo principal deste trabalho é relatar um caso sobre um adenocarcinoma ductal invasor que originou metástase gástrica em paciente, levando a um quadro de abdome agudo obstrutivo. Abrindo espaço para a discussão sobre as condutas a serem tomadas frente a suspeita dessa patologia, considerada incomum e de alta letalidade. 


\section{DETALHAMENTO DO CASO}

Paciente do sexo feminino, 51 anos, casada, natural de Minas Gerais. Deu entrada em serviço terciário Santa Casa de Misericórdia de Belo Horizonte no dia 17/05/19, encaminhada da Unidade de Pronto Atendimento, com queixa de dor e distensão abdominal há duas semanas, o quadro inicial se apresentava com diarréia, seguido de ausência de evacuações e mínima eliminação de flatos. Ao exame físico o abdome apresentava-se globoso, distendido, com alças intestinais visíveis em diástase do músculo reto abdominal. Sem demais alterações ao exame físico relevantes no momento. Ao realizar uma radiografia de tórax foi demostrado um sinal de empilhamento de moedas em intestino delgado. Prosseguiu-se o caso com passagem de sonda nasoentérica com drenagem de secreção enteral. Os exames laboratoriais solicitados na propedêutica de abdome agudo demonstravam apenas discreta hipocalemia $(3,3 \mathrm{mEq} / \mathrm{L})$ como alteração.

Paciente com histórico de câncer ductal invasor de mama hormônio dependente e mastectomia R0 em 2016 à direita, com necessidade de quimio e radioterapia adjuvante. Em uso regular de tamoxifeno, que se enquadra na categoria de modulador seletivo do receptor de estrogênio (SERM's), sendo ele um estrogênio sintético que se liga aos receptores presentes na mama e não induz a liberação de RNA mensageiro, além de impedir a ocupação do receptor pelo estrogênio natural. Sem outras comorbidades.

Diante do quadro, foi solicitado tomografia de abdome superior, inferior e pelve em caráter de urgência no dia 17/05/19, que demonstrava espessamento difuso inespecífico das paredes gástricas do estômago, sem pontos de obstrução mecânica definidos. Então, foi feita a opção por manter a conduta conservadora.

Paciente evoluiu no dia 22/05/19 com vômitos refratários a medicamentos, em grande volume, aumento da distensão abdominal e dor importante. Novos exames de imagem mostraram espessamento e densificação do grande omento e foco de espessamento irregular peritoneal associado a ascite, com possibilidade de acometimento neoplásico secundário.

Em vista da manutenção dos sintomas e dos achados da tomografia, foi realizada uma endoscopia digestiva alta (EDA) com biópsia, na qual se observou pangastriste enantematosa com edema acentuado no antro e mucosa endurecida, com possibilidade de linite plástica ou síndrome de Menetrier. $\mathrm{O}$ exame anatomopatológico sugeriu neoplasia epitelial maligna, com possibilidade de carcinoma mamário metastático, com necessidade de estudo imuno-histoquímico para diagnóstico definitivo.

O relatório imuno-histoquímico demonstrou a expressão para CK7 e de receptor de estrógeno. Neste contexto, compatível com neoplasia metastática de possível sítio primitivo em mama. Como medidas terapêuticas, ofereceu-se a paciente a jejunostomia, para manutenção do estado nutricional, que poderia ser comprometida em eventual obstrução do trânsito intestinal e suporte paliativo.

A paciente, todavia, recusou-se a demais tratamentos, solicitando alta hospitalar, mediante a termo de alta a pedido contra recomendações médicas. O seguimento foi perdido, pois paciente não retornou ao serviço terciário.

\section{DISCUSSÃO}

No caso relatado, a paciente apresentava sintomas de abdome agudo obstrutivo, como distensão abdominal, diarreia paroxística seguida de ausência de evacuações e presença de radiografia de tórax sugestiva de obstrução de delgado. Tais condições clínicas levam a necessidade de solicitação de propedêutica mais ampla e adequada para investigação dessa síndrome (exames laboratoriais, endoscopia digestiva alta com biópsia e tomografia computadorizada).

A ausência de pontos de obstrução mecânica e a presença de ascite e espessamento do omento na tomografia computadorizada sugeriam acometimento neoplásico devendo sempre ser investigada mais detalhadamente.

Como exames anteriores apresentavam alterações inespecíficas no estômago, foi realizada uma endoscopia digestiva alta. Apesar de um sinal específico, a ausência de massa palpável e endurecida em região epigástrica não poderia afastar a hipótese diagnóstica de neoplasia gástrica. A linite plástica pode não ser diagnosticada durante a EDA, pois as células neoplásicas se espalham pela submucosa, poupando a 
mucosa, levando a um espessamento das pregas. Por ser um exame examinador dependente, esse tipo de alteração pode ser julgado como uma gastrite crônica ou aguda. Todavia, o encontro de pregas gástricas espessas, com mucosa nodular e lesões em aspecto vermelho carmim são sugestivas de linite. Neste caso, alguns diagnósticos diferenciais são Síndrome de Ménétrier, amiloidose, linfoma e hiperplasia linfóide (WINDHAM TC, et al., 2002; CARPENTIER S, et al.,1992; HAMY A, et al.,1999).

O estudo anatomopatológico apontava a possível relação com o câncer de mama prévio, cujo tipo histológico mais comum é o lobular invasor, o qual é normalmente diagnosticado em paciente mais velhas do que as portadoras de adenocarcinoma ductal invasor (SANTOS T, et al., 2020). A imuno-histoquímica com presença do padrão $\mathrm{Ck} 7$ positivo e Ck20 negativo e de receptores estrogênicos confirmou a metástase gástrica com sítio primário na mama. Apesar de evento incomum, a metástase gástrica deve ser considerada em pacientes com histórico de câncer de mama que apresentem sintomas gastrointestinais, em especial os dispépticos.

A alta morbimortalidade desses pacientes faz com que essa condição seja considerada um dos principais diagnósticos diferenciais, exigindo propedêutica adequada e rápida, uma vez que a doença é agressiva e não se limita a parede gástrica, como evidenciado nos exames de estadiamento, ocasionando prognóstico reservado.

Em relação ao diagnóstico de linite plástica, se trata de um tumor infiltrante que constitui até $5 \%$ dos casos de tumores gástricos, sendo mais frequente em pacientes do sexo feminino, de origem asiática (populações coreanas, chinesas, tailandesas e japonesas) e com menos de 40 anos, as vezes em pacientes muito jovens (20 a 25 anos de idade) (DUCREUX M, et al., 1998). Eles afetam grande parte da parede gástrica ou até mesmo a parede globalmente. Seu crescimento ocorre de maneira profunda, podendo apresentar mucosas íntegras ou com pregas distorcidas, entretanto as paredes se tornam pouco complacente à insuflação alem de apresentarem pouco peristaltismo.

A linite plástica histologicamente se caracteriza por aumento do tecido conjuntivo associado à proliferação glandular maligna de células independentes, do tipo células em anel de sinete, e um estroma fibroso que acomete todas as camadas gástricas, normalmente respeitando a mucosa, sendo classificado como Bormann 4. Devido a esse tipo de apresentação, a biópsia endoscópica convencional geralmente não é capaz diagnosticar a linite plástica, o que acarreta dificuldade ao diagnóstico e mau prognóstico, sendo necessária a realização de uma macrobiópsia ou biópsia dirigida por ultrassom endoscópico (CARPENTIER S, et al., 1992). Para que se possa avaliar a extensão local, a tomografia computadorizada e a ecografia endoscópica podem ser úteis, embora o custo de tais exames e sua disponibilidade sejam um fator dificultador.

O prognóstico desse tipo de tumor é ruim, e freqüentemente invade os linfonodos regionais e o peritônio, condição conhecida como carcinomatose peritoneal (SANTOS T, et al., 2020). É descrito da mesma forma, o envolvimento intestinal metastático com comportamento infiltrativo semelhante o que faz com que a cirurgia com intenção curativa só seja possivel em aproximadamente 20 a $25 \%$ dos casos. Para o tratamento da linite gástrica sem envolvimento peritoneal é recomendada a ressecção total, com sobrevida após gastrectomia total em um ano de $50 \%$ e em sete anos de $8 \%$ (DE ARRUDA J, et al., 2020).

Nesse tipo de cirurgia, retiram-se o estomâgo, os linfonodos e o omento, e a parte distal do esôfago é anastomosada à parte proximal do jejuno, reconstituindo-se assim o trânsito intestinal por meio da anastomose em Y de Roux. A linfadectomia a D2 é o mais comum, quando se retiram todos os linfonodos que circundam a lesão e os das artérias próximais. Em relação a margem cirúrgica, esta deve ser de pelo menos $10 \mathrm{~cm}$. Na presença de ascite, a análise do líquido também está indicada. Existe consenso entre os cirurgiões quanto a esplenectomia, devendo essa ser realizada.

As principais complicações precoces desse procedimento são a atelectasia, a pneumonia e a insuficiência respiratória. Já as tardias são as fístulas da anastomose esôfago-jejunal, necessitando de jejum oral, nutrição parenteral e antibioticoterapia para seu tratamento, as estenoses de anastomose e os efeitos gastroentestinais como diarreia, dumping precoce e tardio e síndromes disabsortivas. No entanto, muitos cirurgiões consideram a presença de linite plástica uma contra-indicação para a ressecção curativa (NGUYEN S, et al., 2017). 
O acompanhamento pós operatório em relação a qualidade de vida do paciente pode ser realizado por meio da escala de GOOSS (Gastric Outlet Obstruction Scoring System), proposta por Adler et al. Metástases para fígado e peritônio, aumento de fosfatase alcalina $(<100)$ e redução da capacidade funcional avaliada por meio da escala de Performance Status sugerem uma pior evolução clínica (DE ARRUDA J, et al., 2020).

Cirurgias menos agressivas, como a gastrectomia subtotal só podem ser realizadas em pacientes com tumores gástricos restritos ao antro, sem acometimento linfonodal difuso. Não existem diretrizes consensuais que estabeleçam a realização da quimioterapia, obtendo resultados variáveis em termos de sobrevida. E a radioterapia adjuvante após a excisão completa do tumor primário é menos efetiva quando se trata da linite.

Contudo, se realizadas as cirurgias com intuito curativo, o melhor exame a ser realizado é o PET/CT com fluordesoxiglicose (FDG). Este ainda não tem seu valor pré-operatório bem estabelecido. Contudo, alguns estudos apontam que tumores com alta avidez pela FDG tiveram pior prognóstico em comparação com os de baixa avidez (ANDREOLLO N, et al., 2017; TAAL BG, et al., 2000; MCLEMORE EC, et al., 2005).

Ainda que a evolução clínica da doença leve a um quadro de emagrecimento e desnutrição, o suporte nutricional faz-se necessário de maneira precoce, sempre por via enteral, quando possível. A desnutrição deve ser avaliada por métodos antropométricos e bioquímicos, principalmente à admissão. A avaliação do estado nutricional pode ainda ser baseada em escalas já validadas, como a Avaliação Subjetiva Global Produzida pelo Próprio Paciente (asG-PPP). A redução do peso por si só já é um indicativo de aumento de complicações e piora da evolução (LIMA LC, et al., 2018).

Outro ponto importante a ser abordado são os cuidados paliativos. O máximo de conforto deve ser proporcionado a esse paciente, tendo em vista tanto a nutrição, como a analgesia, o cuidado psicoterapêutico e o contato humanizado com a equipe multiprofissional, sendo pregado o princípio da ortotanásia (GOMES ALZ e OTHERO MB, 2016).

A metástase gástrica, especialmente na forma histológica e morfológica de linite plástica, é um evento raro, quando o sítio primário é a mama. Todavia, deve ser considerada naqueles pacientes com histórico de câncer de mama com sintomas dispépticos. Perante o quadro de abdome agudo, deve-se analisar com cautela a hipótese diagnóstica de obstrução por metástases nesses pacientes. Normalmente, exames complementares são necessários para definir condutas. O prognóstico é reservado, visto as limitações no tratamento da linite.

\section{REFERÊNCIAS}

1. ABID A, et al. Breast cancer metastasis to the Gl tract may mimic primary gastric cancer. J Clin Oncol. 2013; 31(7): 106-7.

2. ANDREOLLO N, et al. Complicações pós-operatórias após gastrectomia total no câncer gástrico: análise de 300 doentes. ABCD, arq. Bras. cir. dig., São Paulo, 2011; 24(2): 126-130.

3. ARAÚJO LH, et al. Metástase gástrica de câncer de mama: relato de caso e revisão de literatura. Revista Brasileira de Cancerologia, 2007; 53(3): 365-368.

4. CARPENTIER S, et al. Linite gastrique metastatique. Diagnostic et surveillance par echo-endoscopie au cours du tratment. Gastroenterol Clin Biol 1992; 16: 898-900.

5. CIULLA A, et al. Gastric metastases originating from occult breast lobular carcinoma: diagnostic and therapeutic problems. World J SurgOncol. 2008; 6: 78.

6. DE ARRUDA J, et al. Bipartição gástrica para tratamento paliativo de câncer de estômago avançado: relato de caso. Rev Med (São Paulo), 2020; 99(2): 202-8.

7. DUCREUX M, et al. La linite gastrique atteint desmala des jeunes et repond peu a la chimiotherpie. Gastroenterol Clin Biol 1998; 22/A: 159.

8. GOMES, ALZ, OTHERO MB. Cuidados paliativos. Estudos avançados, 2016; 30(88): 155-166.

9. HAMY A, et al. Study of survival and prognostic factors in patients undergoing resection for gastric linitis plastica: a review of 86 cases. IntSurg 1999; 84: 337.

10. HARA F, et al. Metastatic breast cancer to the stomach resembling early gastric cancer. Case Rep Oncol. 2010; 3(2): 142-7.

11. JONES GE, et al. Breast cancer metastasis to the stomach may mimic primary gastric cancer: report of two cases and review of literature. World J Surg Oncol. 2007; 5: 75.

12. JUCA PC. Análise comparativa e controlada entre os perfis moleculares imuno-histoquímicos do carcinoma mamário, da metástase gástrica de origem mamária e do adenocarcinoma gástrico primário, 2016. 
13. LIBÂNIO D, et al. Metástases Gástricas de Carcinoma da Mama após 20 Anos. GE-Portuguese Journal of Gastroenterology, 2018; 25(2): 99-101.

14. LIMA LC, et al. Manejo Nutricional em Paciente com Metástase Gástrica de Câncer de Mama: um Relato de Caso. Revista Brasileira de Cancerologia 2018; 64(1): 107-112.

15. MCLEMORE EC, et al. Breast cancer: presentation and intervention in women with gastrointestinal metastasis and carcinomatosis. Ann Surg Oncol. 2005; 12(11): 886-94

16. NGUYEN S, et al. Lymphedema of the lower limbs: Initial manifestation of gastric linitis plastica. In: Annales de Dermatologie et de Venereologie. 2017; 530-535.

17. ORTEGA M, et al. Linitis plástica intestinal: metástasis tardía de adenocarcinoma gástrico enanillo de sello. Cir Esp 2006; 80(3): 171-3.

18. RODRIGUES MV, et al. Metástases gástricas de câncer de mama: quando a gastrectomia está indicada? ABCD. Arquivos Brasileiros de Cirurgia Digestiva (São Paulo), 2016; 29(2): 86-89.

19. SANTOS T, et al. Achados clínicos em pacientes com dor abdominal aguda submetidos a tomografia computadorizada em um serviço de urgência. Revista Eletrônica Acervo Científico, 2020; 8: e3069-e3069.

20. TAAL BG, et al. Clinical presentation, endoscopic features, and treatment of gastric metástases from breast carcinoma. Cancer. 2000; 89: 2214-221.

21. WINDHAM TC, et al. Adenocarcinoma of the stomach in patients age 35 years and younger: no impact of early diagnosis on survival outcome. J SurgOncol, 2002; 81: 118. 